\section{Estrogenic Effects of Fluorotelomer Alcohols for Human Estrogen Receptor Isoforms $\alpha$ and $\beta$ in Vitro}

\author{
Hiroshi IshibashI, ${ }^{a}$ Haruna IshidA, ${ }^{a}$ \\ Munekazu MatsuoKa, ${ }^{a}$ Nobuaki Tominaga, ${ }^{b}$ and \\ Koji Arizono*,a
}

${ }^{a}$ Faculty of Environmental and Symbiotic Sciences, Prefectural University of Kumamoto; 3-1-100 Tsukide, Kumamoto 862-8502, Japan: and ${ }^{b}$ Department of Chemical and Biological Engineering, Ariake National College of Technology; 150 Higashihagio-machi, Omuta, Fukuoka 836-8585, Japan.

Received March 3, 2007; accepted April 15, 2007; published online April 17, 2007

\begin{abstract}
The present study demonstrates the estrogenic effects of fluorotelomer alcohols (FTOHs). In a yeast two-hybrid assay, treatment with $1 H, 1 H, 2 H, 2 H$-perfluorooctan-1-ol (6:2 FTOH), $1 H, 1 H, 2 H, 2 H$-perfluoro-decan-1-ol (8:2 FTOH) and 2,2,3,3,4,$4,5,5,6,6,7,7,8,8,9,9,10,10,10$-nonadecafluoro-1-decanol (NFDH) showed a dose-dependent interaction between the human estrogen receptor ( $h E R$ ) isoforms $h E R \alpha$ or $h E R \beta$ ligand-binding domain and coactivator TIF2, whereas there were no estrogenic effects of perfluorooctane sulfonate (PFOS) and perfluorooctanoic acid (PFOA) for these hERs. The estrogenic effects of FTOHs on $\mathrm{hER} \alpha$ were higher than those on $\mathrm{hER} \beta$, indicating a differential responsiveness of hERs to FTOHs. The relative ranks of tested chemicals on the estrogenic effects for $h E R \alpha$ and hER $\beta$ descended in the order of estradiol-17 $\beta \ggg 6: 2$ FTOH $>$ NFDH $>$ 8:2 FTOH. These results suggest that certain FTOHs including 6:2 FTOH, 8:2 FTOH and NFDH interact with hER isoforms $\alpha$ and $\beta$ in vitro. Further studies are necessary to investigate contamination levels, potential biological effects and the risks of these compounds on human health.
\end{abstract}

Key words human estrogen receptor $\alpha$; human estrogen receptor $\beta$; fluorotelomer alcohols; estrogenic effect

Perfluorochemicals (PFCs) such as perfluorooctane sulfonate (PFOS) and perfluorooctanoic acid (PFOA) are widespread contaminants that have been detected in wildlife and humans. ${ }^{1,2)}$ Various studies have demonstrated the potential maternal and developmental toxicity of these compounds in experimental animals. ${ }^{3)}$ Fluorotelomer alcohols (FTOHs) such as 6:2, 8:2 and 10:2 FTOH are classes of compounds recently identified as potential contaminant sources of PFCs, including PFOA, in the environment. Their presence is particularly noted in the atmospheric environment. ${ }^{4,5)}$

Limited information is currently available on the toxicological effects and the risks of FTOHs in experimental animals, although these compounds are metabolically converted to PFOA, associated with the induction of hepatic peroxisome proliferation and acyl-CoA oxidase (ACOX) activity. ${ }^{6}$ Interestingly, a recent study showed the proliferation-promoting capacity of 6:2 FTOH and 8:2 FTOH with an E-screen assay of MCF-7 cell lines. ${ }^{7}$ However, in that study there was no information on the interaction of FTOHs toward human estrogen receptor $\alpha(\mathrm{hER} \alpha)$ or $\beta$ (hER $\beta)$. To evaluate the potential risks of FTOHs in humans, it is important to investigate estrogenic effects at the molecular level, because PFCs including PFOA, which are degraded from FTOHs, are wide- spread contaminants that have been detected in human blood samples. ${ }^{2)}$ Therefore, in this study, we investigated the estrogenic effects of FTOHs for the $\mathrm{hER} \alpha$ or $\mathrm{hER} \beta$ using a yeast two-hybrid assay.

\section{MATERIALS AND METHODS}

Test Chemicals 6:2 FTOH $(1 H, 1 H, 2 H, 2 H$-perfluorooctan-1-ol, Alfa Aesar, MA, U.S.A.), 8:2 FTOH (1H,1H,2H,$2 H$-perfluoro-decan-1-ol, Alfa Aesar), NFDH (2,2,3,3,4,4,5,5,6,6,7,7,8,8,9,9,10,10,10-nonadecafluoro-1-decanol, Wako Pure Chemical Industries, Ltd., Tokyo, Japan), PFOS (Avocado Research Chemicals Ltd., Lancashire, U.K.), PFOA (Tokyo Chemical Industry Co., Ltd., Tokyo, Japan) and E2 (estradiol-17 $\beta$, Sigma, St. Louis, MO, U.S.A.), as a positive control substance, were used in this study. These reagents were dissolved in DMSO (dimethyl sulfoxide, Wako Pure Chemical Industries, Ltd.) to prepare test solutions.

Yeast Two-Hybrid Assay The assay for determining the estrogenic activity of test chemicals was performed as previously described. ${ }^{8)}$ Yeast cells (Saccharomyces cervisiae Y190) were modified by incorporation of hER isoforms (hER $\alpha$ or hER $\beta$ ), an expression plasmid of the coactivator TIF2, and a $\beta$-galactosidase expression reporter in a yeast two-hybrid assay. ${ }^{9)}$ This assay system employs the interaction between the hER $\alpha$ or hER $\beta$ ligand binding domain and TIF2. A previous study using this assay system demonstrated that the E2-dependent interaction of ER with TIF2 was higher sensitivity than that with various coactivators such as SRC1, RIP140, TIF1, p300 and CBP. ${ }^{9)}$

A test solution was incubated $\left(30^{\circ} \mathrm{C}, 4 \mathrm{~h}\right)$ with yeast cells in a 96-well microplate (SUMILON, Sumitomo Bakelite, Japan) that had been pre-incubated $\left(30^{\circ} \mathrm{C}\right.$, overnight) in modified synthetic dropout (SD) medium lacking tryptophan and leucine. A mix solution for inducing chemiluminescence and for enzymatic digestion (Zymolyase 20T) was added to the yeast cells followed by a light emission accelerator solution. The chemiluminescence produced by released $\beta$-galactosidase was measured with a 96-well plate luminometer (Luminescencer-JNR AB2100, ATTO Bio-Instrument, Tokyo, Japan).

The estrogenic activity of test compounds was recorded as the $\mathrm{EC}_{\times 10}$ which was defined as the concentration of test solution producing a chemiluminescent signal $10 \times$ that of the blank control. The inverse of the obtained $\mathrm{EC}_{\times 10}$ values of E2 was set to 100 . Similar procedures were performed with other samples to calculate the E2 relative activity.

\section{RESULTS AND DISCUSSION}

In this study, we investigated the estrogenic effects of FTOHs for the $\mathrm{hER} \alpha$ and $\mathrm{hER} \beta$ using a yeast two-hybrid assay. Treatments with 6:2 FTOH, 8:2 FTOH and NFDH dose-dependently induced hER-mediated transcriptional activity with interaction between the hER $\alpha$ or $\mathrm{hER} \beta$ ligandbinding domain and TIF2, whereas no activation of hERs was observed when treated with PFOS and PFOA (Fig. 1). Maras et $a l^{7}{ }^{7}$ recently demonstrated the proliferation-promoting capacity of 6:2 FTOH and 8:2 FTOH with an Escreen assay of MCF-7 cell lines, but there was no informa- 


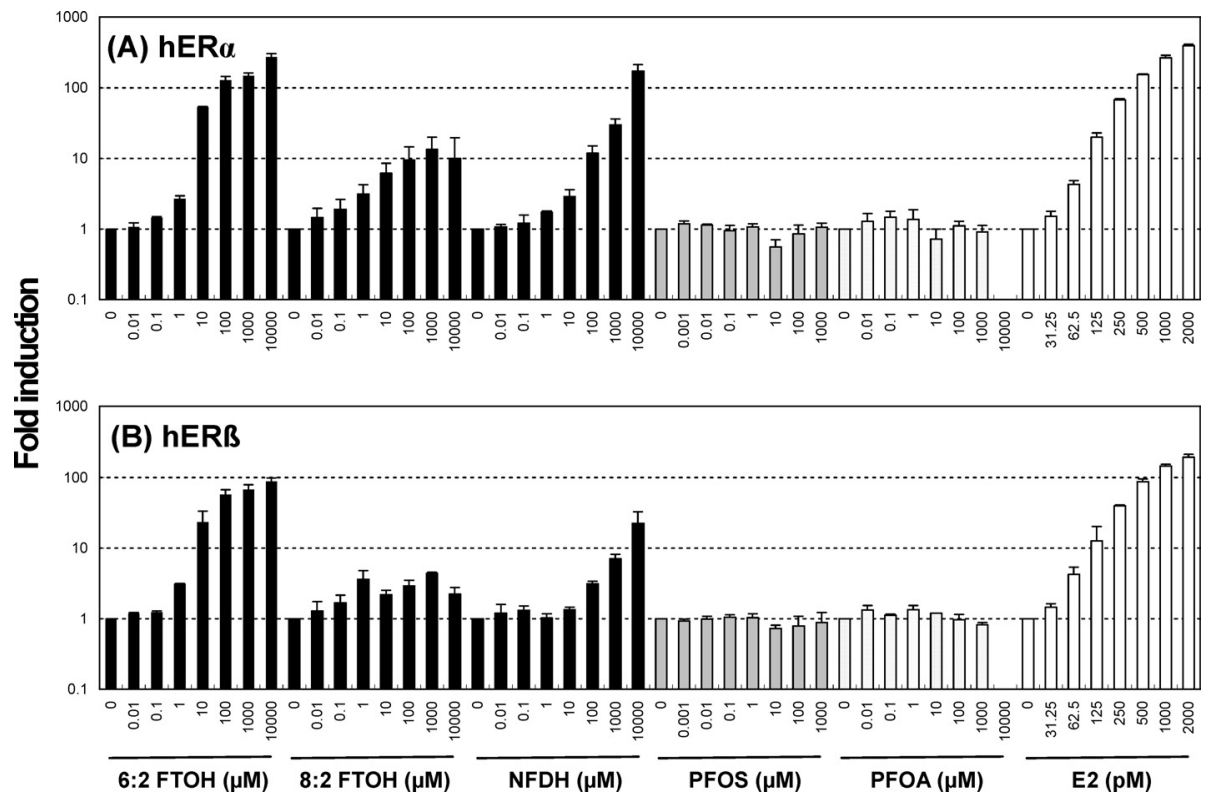

Fig. 1. Estrogenic Effects of FTOHs (6:2 FTOH, 8:2 FTOH and NFDH), Perfluorooctane Sulfonate (PFOS), Perfluorooctanoic Acid (PFOA) and Estradiol$17 \beta$ (E2) for Human Estrogen Receptor $\alpha(\mathrm{hER} \alpha, \mathrm{A})$ and $\beta(\mathrm{hER} \beta, \mathrm{B})$ Using the Yeast Two-Hybrid Assay

Data are presented as the mean and vertical error bars represent standard deviation $(n=3)$.

Table 1. Comparison of $\mathrm{EC}_{\times 10}$ Values and Relative Estrogenic Activities of FTOHs (6:2 FTOH, 8:2 FTOH and NFDH) and Estradiol-17 $\beta$ (E2) for Human Estrogen Receptor $\alpha(\mathrm{hER} \alpha)$ and $\beta(\mathrm{hER} \beta)$ Using the Yeast TwoHybrid Assay

\begin{tabular}{|c|c|c|c|c|}
\hline \multirow{2}{*}{ Chemical } & \multicolumn{2}{|c|}{$\operatorname{hER} \alpha$} & \multicolumn{2}{|c|}{$\mathrm{hER} \beta$} \\
\hline & $\mathrm{EC}_{\times 10}(\mu \mathrm{M})$ & $\begin{array}{l}\text { Relative } \\
\text { activity }\end{array}$ & $\mathrm{EC}_{\times 10}(\mu \mathrm{M})$ & $\begin{array}{l}\text { Relative } \\
\text { activity }\end{array}$ \\
\hline E2 & $9.0 \times 10^{-5}$ & 100 & $1.0 \times 10^{-4}$ & 100 \\
\hline $6: 2 \mathrm{FTOH}$ & 2.3 & $3.7 \times 10^{-3}$ & 4.1 & $2.5 \times 10^{-3}$ \\
\hline $8: 2 \mathrm{FTOH}$ & 545 & $1.6 \times 10^{-5}$ & $>10000$ & Not calculated \\
\hline NFDH & 86 & $1.0 \times 10^{-4}$ & 1562 & $6.4 \times 10^{-6}$ \\
\hline
\end{tabular}

The estrogenic activity of test compounds for hERs was recorded as the $\mathrm{EC}_{\times 10}$ which was defined as the concentration of test solution producing a chemiluminescent signal $10 \times$ that of the blank control. The inverse of the obtained $\mathrm{EC}_{\times 10}$ values of $\mathrm{E} 2$ was set to 100 . Similar procedures were carried out with other samples to calculate the relative E2 activity.

tion on the interaction of FTOHs toward hER isoforms. To our knowledge, this is the first report on the estrogenic effects of FTOHs such as 6:2 FTOH, 8:2 FTOH and NFDH for the hER isoforms in vitro.

For hER $\alpha$ activation by FTOHs, the $\mathrm{EC}_{\times 10}$ values of $6: 2$ FTOH, 8:2 FTOH and NFDH were estimated to be 2.3, 545 and $86 \mu \mathrm{M}$, respectively (Table 1 ). The $\mathrm{EC}_{\times 10}$ values of $6: 2$ FTOH and NFDH on the activation of $h E R \beta$ were estimated to be 4.1 and $1562 \mu \mathrm{M}$, respectively (Table 1). Although activation of $\mathrm{hER} \beta$ by treatment with 8:2 FTOH was observed, the assay system used in this study could not be used to calculate $\mathrm{EC}_{\times 10}$ values because of the low activity of $\beta$-galactosidase expression in the yeast two-hybrid assay (Table 1). Neither study reported effective concentrations for the effect of FTOHs and with the different cell types and detection systems, incubation times and positive controls, it is not easy to compare directly the data with that from previous studies. ${ }^{7}$ In the present study, the overall $\mathrm{EC}_{\times 10}$ values of FTOHs on $\operatorname{hER} \alpha$ activation were lower than those for $\operatorname{hER} \beta$, indicating a differential responsiveness of hERs to FTOHs. The relative ranks of tested chemicals on the estrogenic effects for hERs descended in the order of estradiol-17 $\beta \ggg 6: 2$ FTOH $>$ NFDH $>8: 2$ FTOH. These results suggest that FTOHs including 6:2 FTOH, 8:2 FTOH and NFDH interact with hER isoforms $\alpha$ and $\beta$ in vitro, and that certain FTOHs may be associated with potential biological effects via the ER signaling pathway in humans.

Although the present study demonstrates the estrogenic effects of FTOHs for hER isoforms $\mathrm{hER} \alpha$ and $\mathrm{hER} \beta$ in vitro, the biological effects of these compounds on humans are unclear. In experimental animals such as rats, 8:2 FTOH was metabolically converted to PFOA associated with the induction of hepatic peroxisome proliferation and ACOX activity. ${ }^{6}$ ) These compounds were observed at concentrations ranging from 7 to $196 \mathrm{pg} / \mathrm{m}^{3}$ and from 11 to $165 \mathrm{pg} / \mathrm{m}^{3}$ in the troposphere. ${ }^{4,5)}$ There is no information about the contamination levels of FTOHs in the human body; therefore, further studies are necessary to investigate the contamination level, potential biological effects and the risks of these compounds.

\section{REFERENCES}

1) Giesy J. P., Kannan K., Environ. Sci. Technol., 35, 1339-1342 (2001).

2) Kannan K., Corsolini S., Falandysz J., Fillmann G., Kumar K. S., Loganathan B. G., Mohd M. A., Olivero J., Van Wouwe N., Yang J. H., Aldoust K. M., Environ. Sci. Technol., 38, 4489-4495 (2004).

3) Kennedy G. L., Jr., Butenhoff J. L., Olsen G. W., O’Connor J. C., Seacat A. M., Perkins R. G., Biegel L. B., Murphy S. R., Farrar D. G., Crit. Rev. Toxicol., 34, 351-384 (2004).

4) Martin J. W., Muir D. C., Moody C. A., Ellis D. A., Kwan W. C., Solomon K. R., Mabury S. A., Anal. Chem., 74, 584-590 (2002).

5) Stock N. L., Lau F. K., Ellis D. A., Martin J. W., Muir D. C., Mabury S. A., Environ. Sci. Technol., 38, 991-996 (2004).

6) Kudo N., Iwase Y., Okayachi H., Yamakawa Y., Kawashima Y., Toxicol. Sci., 86, 231-238 (2005).

7) Maras M., Vanparys C., Muylle F., Robbens J., Berger U., Barber J. L., Blust R., De Coen W., Environ. Health Perspect., 114, 100-105 (2006).

8) Miyahara M., Ishibashi H., Inudo M., Nishijima H., Iguchi T., Guillette L. J., Jr., Arizono K., J. Health Sci., 49, 1-11 (2003).

9) Nishikawa J., Saito K., Goto J., Dakeyama F., Matsuo M., Nishihara T., Toxicol. Appl. Pharmacol., 154, 76-83 (1999). 